INRA Prod. Anim.,

2012, 25 (3), 233-244

\title{
Génétique des caprins laitiers
}

\author{
E. MANFREDI', T. ADNØY2 \\ ${ }^{1}$ INRA, UR631 Station d'Amélioration Génétique des Animaux, \\ F-31326 Castanet-Tolosan, France \\ 2 Norwegian University of Life Sciences, Department of Animal and Aquacultural Sciences, \\ P.O. Box 5003, N-1432 As, Norway \\ Courriel : eduardo.manfredi@toulouse.inra.fr
}

\begin{abstract}
Animal de subsistance ou véritable usine de production laitière ? La chèvre, légendaire nourricière des dieux de l'antiquité, continue de surprendre par sa remarquable plasticité lui permettant de s'adapter à des systèmes de production très variables. En amélioration génétique, on retrouve aussi des situations très diverses allant de la sélection artisanale à des schémas de sélection faisant intervenir des outils puissants pour l'évaluation génétique et la reproduction. Nous discutons ici les programmes de sélection actuels et leurs perspectives.
\end{abstract}

Avec plus de 900 millions de têtes en 2010, le cheptel caprin mondial représente désormais respectivement 64,85 et $95 \%$ des cheptels bovins, ovins et porcins (http://faostat.fao.org). Les caprins sont exploités pour la production de lait, de viande et de phanères dans des conditions d'élevage très variées et sur tous les continents. Capra hircus est la principale sous-espèce domestiquée vers 10500 cal.B.P. ${ }^{1}$ en Asie (Naderi et al 2008). Depuis, une longue sélection artisanale a débouché sur la création de races spécialisées pour la production de lait, de viande ou de phanères, de races à finalité mixte et d'animaux croisés. Ces ressources génétiques, plus de 500 races recensées (Shrestha et Fahmy 2005), sont actuellement exploitées dans plusieurs régions du globe sans que des programmes modernes d'amélioration génétique ne soient nécessairement mis en place. La contribution socio-économique de l'espèce est très variable : dans certaines régions la chèvre est un animal de subsistance («la vache des pauvres») alors qu'elle fournit des produits haut de gamme dans d'autres situations.

Dans cette revue bibliographique, nous nous concentrons sur la génétique caprine orientée principalement vers la production laitière dans le cadre des programmes de sélection basés sur le contrôle de performance, l'indexation et un schéma de création du progrès génétique. La première partie porte sur la variabilité génétique et dresse un état des connaissances sur le déterminisme des caractères d'intérêt zootechnique et la seconde partie est consacrée à l'amélioration génétique. Avant de conclure, nous examinerons les possibilités qu'offrent les approches «omiques» pour les caprins laitiers.

\section{1 / Variabilité génétique des caractères d'intérêt zoo- technique}

La majorité des études génétiques chez la chèvre relèvent encore du modèle dit «polygénique» qui postule l'action additive, homogène et indépendante des nombreux gènes inconnus. C'est le modèle traditionnel de la génétique en zootechnie, basé sur les données des phénotypes et des généalogies, sans recours à des informations moléculaires sur des gènes ou des régions chromosomiques. Rappelons que le génome caprin est seulement en train d'être séquencé et que les outils spécifiques pour les études «omiques» sont encore limités dans l'espèce caprine. Cela n'a pas empêché des études approfondies sur certains gènes caprins que nous discuterons ici. Nous présentons successivement l'état des connaissances du déterminisme génétique des caractères relatifs à la production et aux produits laitiers, à la résistance génétique aux maladies et d'autres caractères, en regroupant à chaque fois les résultats polygéniques (héritabilités et corréla- tions génétiques) et les effets des polymorphismes, des marqueurs génétiques ou des gènes, établis plus récemment.

\section{1 / Production et produits laitiers}

\section{a) La production laitière}

Les caractères de production laitière mesurés dans la routine du contrôle de performance regroupent les quantités de lait, de gras et de protéine, et la composition chimique (taux butyreux et protéique). Le tableau 1 (Belichon et al 1999) donne une vision complète des paramètres polygéniques pour les races Alpine et Saanen françaises. Pour les quantités, les héritabilités sont autour de 0,3 avec des variations assez importantes, moins de 0,2 à plus de 0,5 , rapportées pour d'autres populations dans des conditions de milieu très variables (Kennedy et al 1982, Analla et al 1996). Les corrélations génétiques entre les quantités sont au-dessus de $+0,75$ et la corrélation entre les taux est d'environ $+0,5$ (tableau 1 et Kennedy et al 1982 ; Boichard et al 1989). En revanche, la quantité de lait et les taux sont négativement corrélés alors qu'il y a corrélation positive entre, d'une part, la quantité et le taux de matière grasse et, d'autre part, entre la quantité et le taux de protéines (tableau 1).

Les résultats présentés dans le tableau 1 ont été calculés à partir des productions totales par lactation (lactations d'environ 9 mois). Les valeurs estimées

\footnotetext{
${ }^{1}$ Années calibrées avant le présent.
} 
Tableau 1. Héritabilités (en rouge sur la diagonale), corrélations phénotypiques (au-dessus de la diagonale) et corrélations génétiques ( \pm les erreurs standards correspondantes) des caractéristiques de la production laitière des chèvres de race Alpine et Saanen (Belichon et al 1999).

\begin{tabular}{|l|c|c|c|c|c|}
\hline Race Alpine & QL & QMP & TP & QMG & TB \\
\hline QL & $0,34 \pm 0,002$ & $+0,93$ & $-0,38$ & $+0,85$ & $-0,16$ \\
\hline QMP & $+0,89 \pm 0,006$ & $0,36 \pm 0,002$ & $-0,038$ & $+0,88$ & $+0,01$ \\
\hline TP & $-0,28 \pm 0,024$ & $+0,19 \pm 0,027$ & $0,58 \pm 0,002$ & $-0,11$ & $+0,49$ \\
\hline QMG & $+0,77 \pm 0,012$ & $+0,86 \pm 0,008$ & $+0,14 \pm 0,027$ & $0,37 \pm 0,002$ & $+0,38$ \\
\hline TB & $-0,18 \pm 0,027$ & $+0,11 \pm 0,026$ & $+0,61 \pm 0,016$ & $+0,49 \pm 0,022$ & $0,58 \pm 0,002$ \\
\hline Race Saanen & $0,32 \pm 0,002$ & $+0,95$ & $-0,40$ & $+0,86$ & $-0,12$ \\
\hline QL & $+0,92 \pm 0,005$ & $0,34 \pm 0,02$ & $-0,11$ & $+0,88$ & $-0,01$ \\
\hline QMP & $-0,29 \pm 0,032$ & $+0,10 \pm 0,038$ & $0,50 \pm 0,002$ & $-0,16$ & $+0,41$ \\
\hline TP & $+0,76 \pm 0,014$ & $+0,83 \pm 0,010$ & $+0,08 \pm 0,036$ & $0,40 \pm 0,002$ & $+0,39$ \\
\hline QMG & $-0,10 \pm 0,034$ & $+0,10 \pm 0,032$ & $+0,51 \pm 0,023$ & $+0,56 \pm 0,025$ & $0,60 \pm 0,002$ \\
\hline TB &
\end{tabular}

$\mathrm{QL}=$ Quantité de Lait ; QMP = Quantité de Matière Protéique ; QMG = Quantité de Matière Grasse ; TP = Taux Protéique ; TB = Taux Butyreux.

des héritabilités à partir des données des contrôles laitiers journaliers sont plus faibles. Par exemple, en races Norvégiennes, Holme (2009) rapporte des héritabilités de $0,26,0,43,0,31$ et 0,45 pour respectivement, la quantité de lait, le taux de protéines, le taux de matière grasse et le taux de lactose.

\section{b) Du lait au fromage}

Ces connaissances polygéniques ont été complétées par des études au niveau moléculaire de certains gènes, en particulier celui de la caséine as 1 (locus CSN1S1). Cette protéine est l'une des 4 caséines présentes dans le lait des ruminants $(\alpha s 1-\mathrm{CN}, \alpha s 2-\mathrm{CN}, \beta-\mathrm{CN}$ et $\kappa-\mathrm{CN})$ qui sont essentielles pour la coagulation et la fabrication fromagère. Dès les années 1970, l'INRA a été un protagoniste central des recherches sur les caséines caprines, dans le contexte d'une filière française très orientée vers la production fromagère, mais disposant d'un lait pauvre en protéines coagulables, moins de $30 \mathrm{~g} / \mathrm{kg}$ à l'époque, chez les deux races principales Alpine et Saanen. L'amélioration du rendement fromager était une priorité des organismes de sélection de l'époque, relayée par l'INRA, surtout au Laboratoire de Génétique Biochimique et Cytogénétique $^{2}$ et à la Station d'Amélioration Génétique des Animaux, avec le concours de la Station Caprine de Moissac (coopérative dans la Lozère) et de l'Unité Expérimentale caprine de l'INRA à Bourges. Les protéines coagulables du lait étaient bien décrites dès les années 1970 (Mercier et al 1971, Grosclaude et al 1973) et les polymorphismes génétiques caprins ont été décelés ultérieurement avec des approches de la génétique moléculaire
(Leroux et al 1990, Martin et Grosclaude 1993, Grosclaude et Martin 1997). Actuellement, 16 allèles sont décrits au locus CSN1S1, 7 au locus CSN1S2 (protéine $\alpha s 2-\mathrm{CN}$ ) et 5 au locus CSN2 $(\beta-C N)$. Si dans un lait moyen de chèvres des races Alpine et Saanen, $\alpha$ s $1-\mathrm{CN}$ n'est pas la caséine majoritaire, son locus CSN1S1 est le plus polymorphe. Et ce polymorphisme de structure a des effets quantitatifs sur la synthèse protéique donnant lieu à 4 classes alléliques associées à des quantités de caséine $\alpha$ s 1 qualifiées de «fort», «intermédiaire», «faible» et «nul», cette dernière classe n'exprimant pas la protéine (Grosclaude et al 1987).

Pour estimer au mieux les effets du polymorphisme CSN1S1, des animaux expérimentaux ont été créés dans la Station Caprine de Moissac et l'élevage caprin INRA de Bourges, en accouplant des parents hétérozygotes «fort-faible» ou «fort-nul» de sorte que les descendants «nul-nul» ou «fort-fort» pouvaient provenir d'une même famille avec le même fond génétique (Barbieri et al 1995). Des données en ferme ont été également collectées dans des dispositifs intra-famille (Mahé et al 1994). Les effets des génotypes CSN1S1 ont été comparés quant aux performances des chèvres et de la qualité des produits, laits et fromages. Ainsi, il a été montré que les chèvres porteuses d'allèles forts produisaient, en fermes et en station expérimentale, des laits plus riches en protéines avec une plus grande quantité de matière protéique produite au cours de la lactation (Mahé et al 1994, Barbieri et al 1995). Elles présentaient également une utilisation plus efficace de l'éner- gie des aliments (Schmidely et al 2002). Coté produits, les caractéristiques technologiques des laits «forts» permettaient d'augmenter la fermeté des gels et les rendements fromagers (Remeuf 1993, Vassal et al 1994), et ils avaient une plus grande valeur nutritionnelle (Fevrier et al 2000). En revanche, l'absence de $\alpha$ s1-CN dans le lait des chèvres homozygotes «allèle nul» pouvait être un atout pour la production des laits diététiques (Bevilacqua et al 2001 ) et les allèles faibles pouvaient donner un goût «chèvre» un peu plus fort (Vassal et al 1994). Cette association entre $\alpha \mathrm{s} 1-\mathrm{CN}$ et les caractéristiques organoleptiques est sans doute indirecte, via la composition fine des acides gras, l'activité des lipases ou encore la lipolyse associées aux différents génotypes (Lamberet et al 1996).

Rappelons que l'influence génétique sur la qualité sensorielle des produits laitiers avait été mise en évidence dès les années 1960 chez la chèvre par la comparaison des laits de chèvres des races laitière Norvégienne et Saanen (Skjevdal 1979) et par l'estimation de l'héritabilité du goût "chèvre» à 0,25 (Rønningen 1965) et que des collaborations entre des équipes Norvégiennes et l'INRA se sont mis en place dès les années 1990. Les travaux en cours à l'INRA et cette collaboration ont permis de découvrir un polymorphisme propre aux chèvres Norvégiennes, une délétion ponctuelle dans l'exon $12 \mathrm{du}$ CSN1S1, ayant des effets défavorables sur les taux des caséines et de matière grasse et sur la coagulation des laits (Ådnøy et al 2003). Plus récemment, ces études ont été étendues à l'ensemble des caséines en utilisant des haplotypes

\footnotetext{
2 Génétique Animale et Biologie Intégrative (GABI) depuis 2009.
} 
construits à partir de $39 \mathrm{SNP}^{3}$ qui ont permis de confirmer l'influence majeure de CSN1S1 sur les phénotypes laitiers ainsi que de suggérer des effets de CSN3 $(\kappa-\mathrm{CN})$ sur les taux de matières du lait (Hayes et al 2006).

Ces travaux pionniers ont été ensuite étendus aux polymorphismes des autres caséines, de ses promoteurs (Cosenza et al 2007) et aussi des protéines solubles du lait, dans de nombreuses races (cf. la revue bibliographique d'IbeaghaAwemu et al 2008 sur les gènes à effets majeur chez la chèvre ; la comparaison de génotypes caséines de races caprines aux USA par Maga et al 2009). Citons, par exemple, les études sur la structure du gène de la $\beta$-lactoglobulin (Folch et al 1994) et ses variants (Pena et al 2000, Yahyaoui et al 2000). Mais ce sont surtout les liens entre le polymorphisme CSN1S1 et la composition des Acides Gras (AG) qui continuent à mobiliser les chercheurs. Les génotypes as 1-CN forts et faibles ont des profils d'AG différents (Chilliard et al 2006) et des profils d'expression différents pour 41 gènes dont deux influençant la lipogenèse (Ollier et al 2008). L'hypothèse actuelle ne considère pas qu'une association génétique entre CSN1S1 et d'autres locus agissant sur la composition des $\mathrm{AG}$ soit vraisemblable. Les liens constatés entre la $\alpha s 1-\mathrm{CN}$ et la matière grasse seraient plutôt indirects par des modifications physiologiques et structurelles des cellules épithéliales mammaires associées au polymorphisme CSN1S1 (Chanat et al 1999, Ollier et al 2008). D'une façon plus générale, la variabilité génétique polygénique du contenu en $\mathrm{AG}$ du lait est élevée $\left(\mathrm{h}^{2}=\right.$ 0,42 en chèvres laitières Norvégiennes ; Holme 2009).

\section{c) Morphologie mammaire et aptitude} à la traite

La traite représente la moitié du temps de travail dans l'élevage laitier et elle influence la production laitière, la qualité du lait et la santé des chèvres. L'efficacité de la traite résulte des interactions entre les conditions de traite (niveau de vide, vitesse et taux de pulsation) (Lu et al 1991) et la morphologie et la physiologie de la mamelle (Bruckmaier et al 1994). Les mesures et notes de morphologie mammaire sont héritables, avec des héritabilités élevées $(>0,3)$ pour les mesures sur les trayons (diamètre, longueur, taille) et intermédiaires (autour de 0,25 ) pour les notes subjectives décrivant la suspension de la mamelle (attache arrière, avant-pis, distance plancher-jarret) (Luo et al 1997, Manfredi et al 2001). La morphologie externe a une valeur fonctionnelle mais elle est faiblement corrélée à l'aptitude à la traite, caractère qui a été bien étudié en caprins. L'existence d'un gène à effet majeur sur le «débit $1^{\text {ìre }}$ minute» a été postulée et confirmée par comparaison des demi-sœurs de père (Ricordeau et al 1990, Ilahi et al 2000). Ce gène hypothétique explique la moitié de la variabilité génétique totale du débit $1^{\text {ere }}$ minute (héritabilité totale $>0,5$ vs héritabilité résiduelle polygénique $=0,3$ ). Un allèle favorable et récessif «haut débit (hd)» favoriserait les traites rapides, mais sa localisation et sa fonction restent inconnues. Comme les mamelles des chèvres contiennent des proportions importantes de lait citernal par rapport au lait alvéolaire, les hypothèses courantes privilégient des mécanismes physiologiques responsables de l'ouverture du sphincter au détriment d'un rôle prépondérant de la variabilité dans la régulation de la traite par l'ocytocine (Bruckmaier et al 1994, Marnet et al 2001).

Les relations entre la production, la morphologie mammaire et les caractéristiques de traite sont modérées. Les notes de morphologie sont peu corrélées génétiquement avec la quantité de lait à l'exception notoire de la distance plancher-jarret (corrélation génétique d'environ - 0,5). De même, la corrélation génétique entre quantité de lait et débit $1^{\text {ere }}$ minute est faible $(<0,2$, Ilahi et al 2000) alors que la corrélation phénotypique est d'environ 0,25 (Ilahi et al 1999). Concernant les relations entre la morphologie et l'aptitude à la traite, des associations phénotypiques ont été rapportées, par exemple entre forme et diamètre de trayon et vitesse de traite (Montaldo et Martinez-Lozano 1993, Bruckmaier et al 1994).

Les paramètres génétiques décrits cidessus donnent des tendances générales. Rappelons toutefois que ces paramètres dépendent des modalités des mesures qui varient beaucoup dans les systèmes de collecte d'information de différents pays ou régions. Par exemple, en France et en Norvège l'utilisation des éprouvettes automatiques est de plus en plus répandue alors qu'en Norvège il existe également une note de «facilité de traite» utilisée en routine. Les automatismes peuvent être de type «exhaustifs», avec enregistrement des volumes de lait collectés au cours de la traite (par exemple les éprouvettes développées par l'INRA, Ilahi et al 1999) ou «dérivatifs» qui enregistrent les débits (cas des
Lactocorder ${ }^{\circledR}$ utilisés dans la routine du contrôle laitier). De même, les systèmes de notation de morphologie mammaire peuvent regrouper des mesures (tour de poitrine) et des notes subjectives dans des échelles variables.

\section{2 / Résistance génétique aux maladies}

L'OIE ${ }^{4}$ liste les principales épizooties affectant les caprins : une quinzaine de maladies non spécifiques (brucellose, cowdriose, fièvre Q, paratuberculose...) et des maladies spécifiques des petits ruminants comme la tremblante et l'arthrite/encéphalite caprine. A cela s'ajoutent les maladies métaboliques, les parasitismes et les infections mammaires. Les études génétiques en races traites se sont concentrées en priorité sur des maladies d'importance socioéconomique dont la prévention et le traitement sont difficiles, comme les infections mammaires et la tremblante.

Les mammites subcliniques, décelées par les Comptages de Cellules Somatiques (CCS) mesurés lors du contrôle laitier, ont une héritabilité d'environ 0,2 . Les logarithmes des comptages élémentaires (LSCS), moyennés par lactation, ont des héritabilités moyennes $(0,20$ en race Alpine, 0,24 en race Saanen, Rupp et al 2011) un peu plus élevées que les héritabilités rapportées pour les contrôles élémentaires $(0,14$ en race laitière Norvégienne, Holme 2009). Les CCS ont des corrélations nulles ou faibles avec les caractères de production laitière et de morphologie de la mamelle et des trayons (Holme 2009, Rupp et al 2011). Si ce caractère apparaît pertinent pour la sélection, l'interprétation biologique des valeurs de CCS individuelles très élevées chez les caprins intéresse les généticiens et les pathologistes. A l'INRA, une expérimentation de sélection divergente est en cours pour évaluer les conséquences d'une sélection sur les LSCS et pour élucider les relations entre ce caractère, le statut infectieux et l'aptitude de l'animal à résister à l'infection ( $R$. Rupp communication personnelle, Clément et al 2008).

La tremblante caprine, maladie neurodégénérative de la famille des Encéphalopathies Spongiformes Transmissibles (EST), est l'objet d'un plan de prévention, de contrôle et d'éradication qui inclut l'abattage des animaux et des cheptels atteints. La maladie reste rare en France où environ 1/10 000 des tests annuels est positif, depuis le lancement

\footnotetext{
3 «Single Nucleotide Polymorphism».

4 Organisation mondiale de la santé animale.
} 
$\mathrm{du}$ dispositif national d'épidémiosurveillance mis en place par la DGAL ${ }^{5}$ en 1997. En 2010 par exemple, 7 cas positifs, dont 5 de tremblante «atypique», ont été détectés sur 70905 tests pratiqués (http://agriculture.gouv.fr/tremblante). La protection contre l'infection et la durée d'incubation de la tremblante semblent être contrôlées principalement par le polymorphisme du gène de la protéine prion (PRNP). Les recherches se poursuivent pour confirmer les effets bénéfiques de certains allèles protecteurs contre cette maladie (Barillet et al 2009, Vaccari et al 2009).

\section{3 / Autres caractères}

Les caractères évoqués ci-dessus font partie ou pourraient faire partie des critères de sélection des races traites à court terme. D'autres caractères autour de la croissance, de l'efficacité alimentaire, de la reproduction et des qualités des produits carnés ne seront pas discutés ici. En revanche, nous rappelons l'étude de certains caractères importants dans la sphère de l'insémination artificielle, technologie à la base des schémas de sélection des races traites. En caprins, elle a été couplée souvent avec la congélation de la semence et l'induction/synchronisation d'œstrus (Leboeuf et al 1998, Leboeuf et al 2008). La qualité et la quantité de semence sont des critères importants dans le choix des mâles destinés à l'insémination. Elles ont des héritabilités voisines de 0,30 pour les caractères quantitatifs (nombre et concentration de spermatozoïdes, volume de l'éjaculat). En revanche, la caractérisation de la qualité de la semence par les mesures classiques de motilité ou de pourcentage de spermatozoïdes vivants est délicate (héritabilité inférieure à 0,15) (Manfredi et al 1998, Furstoss et al 2009). Finalement, rappelons que l'absence de cornes a été un critère d'élimination génétique dans le passé car ce caractère est associé à l'intersexualité (Asdell 1944). La mutation PIS (syndrome Motte Intersex ; Vaiman et al 1996, Pailhoux et al 2001) a un effet dominant pour le caractère «absence ou présence de cornes» ( 1 allèle muté entraîne «l'absence de cornes») et un effet récessif sur l'intersexualité, observée chez les individus génétiquement femelles, avec des chromosomes sexuels $\mathrm{XX}$, et porteurs de deux allèles mutés. L'hypothèse actuelle est que la mutation PIS, délétion de $12 \mathrm{~kb}$ dans le chromosome 1 caprin, altère la transcription et l'expression d'au moins deux gènes, Pisrt1 et Fox12, nécessaires pour la différentiation ovarienne chez les individus XX.

\section{2 / Les programmes de sélec- tion}

\section{1 / Généralités}

\section{a) Progrès génétique}

Les différentes composantes d'un programme de sélection sont ses objectifs, le contrôle de performances et des généalogies, l'indexation génétique et le schéma de sélection. Chacune de ces composantes affecte les facteurs du progrès génétique (encadré 1).

L'objectif de la sélection est généralement posé en termes économiques, usuellement comme une somme des valeurs génétiques additives pour plu- sieurs caractères, chacune pondérée par son importance économique. La variabilité d'un objectif agrégat de valeurs génétiques dépend des héritabilités et des corrélations génétiques entre caractères.

Les autres facteurs du progrès génétique varient pour les différents compartiments d'un schéma de sélection (encadré 2). Avec un schéma d'Insémination Artificielle (IA), il convient de distinguer quatre voies de transmission: père-fils, père-fille, mère-fils et mère-fille, chacun ayant son intensité de sélection, sa précision d'index et son intervalle entre générations. Les voies père-fils et mère-fils représentent la sélection sur ancêtres des mâles d'IA, organisée au niveau de la population. La voie père-fille est également organisée au niveau de la population quand le schéma s'appuie à 100\%

\section{Encadré 1. Progrès génétique et composantes du programme de sélection.}

Dans la théorie de la sélection, le progrès génétique $\Delta \mathrm{g}$ est déterminé par l'intensité de sélection $i$, la précision $\rho$ de l'index génétique, la variabilité génétique og et l'intervalle entre générations.

$\Delta g=\sigma g * i * \rho / I G$

$\sigma g$ : variabilité de l'objectif de la sélection

i : intensité de sélection dépendant de la proportion «animaux sélectionnés/animaux candidats»

$\rho$ : précision de l'index génétique utilisé pour calculer le mérite génétique inconnu de chacun des candidats

IG : Intervalle entre Générations

- L'objectif détermine directement $\sigma g$. L'objectif et les critères de sélection évoluent dans le temps : quantité et taux de protéines dans les années 1990, intégration ultérieure de la quantité et du taux de matière grasse et de la morphologie de la mamelle en 2006.

- Le schéma de sélection influence les trois facteurs du progrès : l'intensité de sélection (car il fixe les rapports «nombre de sélectionnés/nombre de candidats»), l'intervalle entre générations et la précision (car il fixe l'âge des candidats au moment de la sélection et en conséquence, la quantité de phénotypes potentiellement disponibles sur le candidat).

- L'acquisition des données joue sur la quantité et la qualité des performances et des généalogies disponibles pour la sélection, donc sur la précision de l'index.

- L'indexation génétique met en jeu des procédures statistiques poussées pour combiner toutes les informations généalogiques et tous les phénotypes nécessaires à l'amélioration de l'objectif. Elle influence la précision des index. La méthode usuellement utilisée est le BLUP ou prédicteur linéaire non biaisé. En France, les index utilisés (Clément et al 2006, Leboeuf et al 2008) sont :

\begin{tabular}{|l|l|c|}
\hline & \multicolumn{1}{|c|}{ Race Alpine } & Race Saanen \\
\hline$I M C$ & $P R M+P L A+Q A A+F A P$ & $P R M+P L A+Q A A+0,5 F A P$ \\
\hline$I C C$ & \multicolumn{2}{|c|}{$M P+0,4 T P+0,2 M G+0,1 T B$} \\
\hline
\end{tabular}

IMC = combinaison d'index morphologiques élémentaires ;

PRM = profil de la mamelle $;$ PLA = distance plancher-jarret ;

$\mathrm{QAA}=$ qualité de l'attache arrière ; FAP = forme de l'arrière pis.

ICC = combinaison d'index élémentaires ;

$\mathrm{MP}=$ quantité de Matière Protéique ; TP = Taux Protéique ;

QMG = quantité de Matière Grasse ; TB = Taux Butyreux.

Le poids de l'IMC dans l'index global est de $44 \%$ en race Saanen et de $33 \%$ en race Alpine.

5 Direction Générale de l'Alimentation. 
Encadré 2. Schéma de sélection des mâles d'Insémination Artificielle (IA).

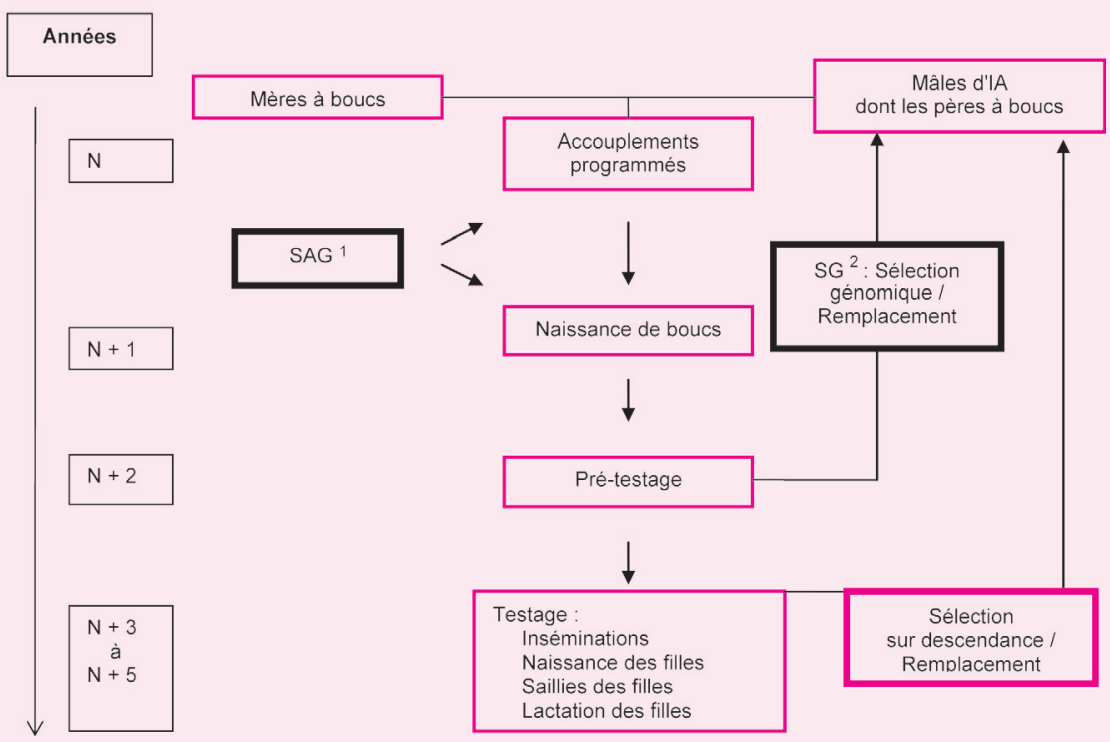

Les rectangles rouges représentent les étapes du schéma actuel de sélection des mâles : accouplements programmés, naissance de mâles un an plus tard, entrée en pré-testage, testage, et sélection/remplacement. L'index polygénique est utilisé pour sélectionner les mères et les pères à boucs, et pour sélectionner 5 ans plus tard les boucs avec les meilleurs tests sur descendance qui remplaceront une fraction des mâles d'IA des générations précédentes.

1 La boîte «SAG» représente la sélection assistée par gènes avec 2 utilisations possibles des génotypes : la planification des accouplements programmés et la sélection intra-fratrie des boucs à la naissance. Seule la deuxième possibilité a été utilisée en pratique pour éliminer des jeunes boucs porteurs des allèles défavorables pour le gène de la caséine. Les étapes du schéma traditionnel sont maintenues, testage inclus.

2 La boîte «SG» montre une utilisation hypothétique de la sélection génomique où le schéma traditionnel est modifié par la suppression du testage sur descendance, une des applications possibles de la SG. Dans ce cas, la sélection et le remplacement des vieux mâles peut être réalisée précocement sans attendre les résultats du testage à $\mathrm{N}+5$.

sur des IA. La voie mère-fille est organisée dans chaque élevage, avec des intensités de sélection souvent faibles. Nous discutons ci-après l'utilisation de la saillie naturelle dans les schémas de sélection caprins.

\section{b) Données moléculaires}

Les données des performances et des généalogies sont désormais complétées par des informations moléculaires issues du génotypage et du séquençage. Les polymorphismes utilisés correspondent à des mutations causales d'action mendélienne ou influençant des caractères complexes, des marqueurs des QTL ${ }^{6}$ (microsatellites, polymorphismes des nucléotides ou «SNP») ou du «nombre des copies des gènes» $\left(\mathrm{CNV}^{7}\right)$.

Les données moléculaires, souvent des génotypages, peuvent être utilisées pour augmenter le progrès génétique par un accroissement de la précision de la sélection $(\rho)$ et de l'intensité de sélection (i), et par la diminution des intervalles entre générations (IG) (encadré 1). L'augmentation de la précision de sélection peut être acquise sans changement du schéma de sélection. Mais c'est surtout en jouant sur l'intensité de sélection et les intervalles entre générations que l'impact peut être plus important. L'atout principal des génotypages est qu'ils sont accessibles très précocement dans la vie d'un animal, et qu'ils sont pérennes, à la différence des phénotypes ou des données transcriptomiques. Dans le cas des races traites, où plusieurs caractères prioritaires sont exprimés seulement chez les femelles, l'information moléculaire autorise la sélection des mâles à leur naissance, avec deux conséquences importantes. D'une part, à coût fixe, en sélectionnant à la nais- sance, il est possible de retenir plus de candidats à la sélection que le nombre habituel de mâles destinés au testage. Cette augmentation de l' «espace de sélection» peut être destinée à améliorer l'intensité de sélection et/ou à intégrer efficacement des nouveaux critères de sélection. D'autre part, la précocité de la mesure permet d'anticiper le moment de la sélection dans la vie de l'animal, et en conséquence, de réduire l'intervalle entre générations.

L'utilisation des typages peut être également orientée vers une réduction des coûts et comme nous allons voir dans la section suivante, il est parfois possible, à l'aide des génotypages, d'allier la réduction des coûts à l'augmentation du progrès génétique.

\section{c) Différentes modalités d'intégration} des outils moléculaires en sélection

La manière d'intégrer les données moléculaires varie selon la nature du déterminisme génétique du caractère et des connaissances acquises sur ce déterminisme. Nous parlerons de Sélection Assistée par Gènes (SAG), quand les mutations causales des phénotypes sont connues, de Sélection Assistée par Marqueurs ou «SAM» (Fernando et Grossman 1989) lorsqu'il s'agit de marqueurs des régions chromosomiques contenant des QTL qui influencent le caractère, et de Sélection Génomique «SG» (Lande et Thompson 1990, Meuwissen et al 2001), lorsqu'un grand nombre de marqueurs est utilisé pour sélectionner les animaux, sans connaissance préalable des effets de chacun des marqueurs. En SAG et SAM, il y a une phase de recherches (souvent avec publication de résultats) sur les mutations causales ou les régions contenant des QTL, alors qu'en SG stricte, les marqueurs sont utilisés sans détection de QTL préalable, avec l'intention de capter les effets des gènes à effet faible que la recherche de QTL n'aurait pas détectés comme significatifs. La méthode suppose que si un grand nombre de marqueurs est bien réparti sur la totalité du génome, au moins quelques marqueurs seront proches, et associés, aux gènes inconnus influençant le phénotype (Meuwissen et al 2001). La validité de ces hypothèses reste du domaine de la recherche, mais la SG peut être justifiée aussi par sa capacité, grâce aux marqueurs, de décrire précisément l'apparentement entre animaux d'une population, pour produire des index plus fiables que le modèle polygénique.

\footnotetext{
${ }^{6}$ QTL est l'acronyme anglais de «Quantitative Trait Loci», soit des groupes de gènes inconnus ayant des effets sur un caractère quantitatif.

${ }^{7} \mathrm{CNV}$ est l'acronyme anglais de «Copy Number Variation», le nombre de copies d'un gène de chaque individu.
} 


\section{d) Sélection génomique en races traites}

En SG, il y a une population de référence dont les individus sont génotypés et phénotypés (population GP, qui servira pour attribuer des valeurs prédictives à chaque génotype marqueur) et une population cible dont seuls les génotypes sont connus (population G). L'index génomique des individus de la population $G$ est calculé en utilisant leurs génotypes et les valeurs prédictives des marqueurs estimées dans la population de référence. Dans l'encadré 3, l'exemple est volontairement simple : 1 seul marqueur et une règle très simple d'attribution de la valeur prédictive (la moyenne arithmétique des phénotypes par génotype). Dans la réalité, il faut attribuer des valeurs prédictives à des milliers de marqueurs d'où la nécessité de disposer d'un nombre important d'individus, quelques milliers, dans la population de référence GP. De même, chaque individu de cette population GP doit être bien caractérisé phénotypiquement (i.e., pour les boucs, avoir un grand nombre de filles). Et les méthodes d'attribution des valeurs prédictives sont nécessairement plus complexes. Il est aussi nécessaire que les associations et les valeurs prédictives des marqueurs trouvées dans la population de référence GP se retrouvent dans la population cible G : c'est le cas quand les deux populations G et GP sont génétiquement proches, par exemple les parents (GP) et leurs descendants (G) (Legarra et al 2008).

La dernière partie de l'encadré 3 illustre trois cas possibles de population de référence permettant de prédire la valeur génétique des jeunes mâles destinés à l'IA. Dans le premier cas, les valeurs prédictives des marqueurs sont estimées en utilisant les résultats des index génétiques des mâles déjà testés sur descendance. L'avantage de ce dispositif est la grande précision des index de ces mâles testés sur plusieurs dizaines de filles, d'où son utilisation répandue en SG des bovins laitiers. Or, en caprins, le nombre de mâles testés par an est d'environ 30 par race, d'où la nécessité de cumuler plusieurs séries de boucs testés sur plusieurs années, pour constituer une population de référence de quelques milliers de boucs testés. Une solution possible est d'inclure des femelles dans la population de référence (deuxième cas dans l'encadré 3 ) pour compléter les effectifs nécessaires, sachant que la précision des index des femelles est plus faible que celle des index des mâles. Le troisième cas de population GP illustré dans l'encadré 3, représente un dispositif de SG appliquée à un caractère qui ne fait pas l'objet du contrôle phénotypique de routine

Encadré 3. Sélection génomique (cas d'école).

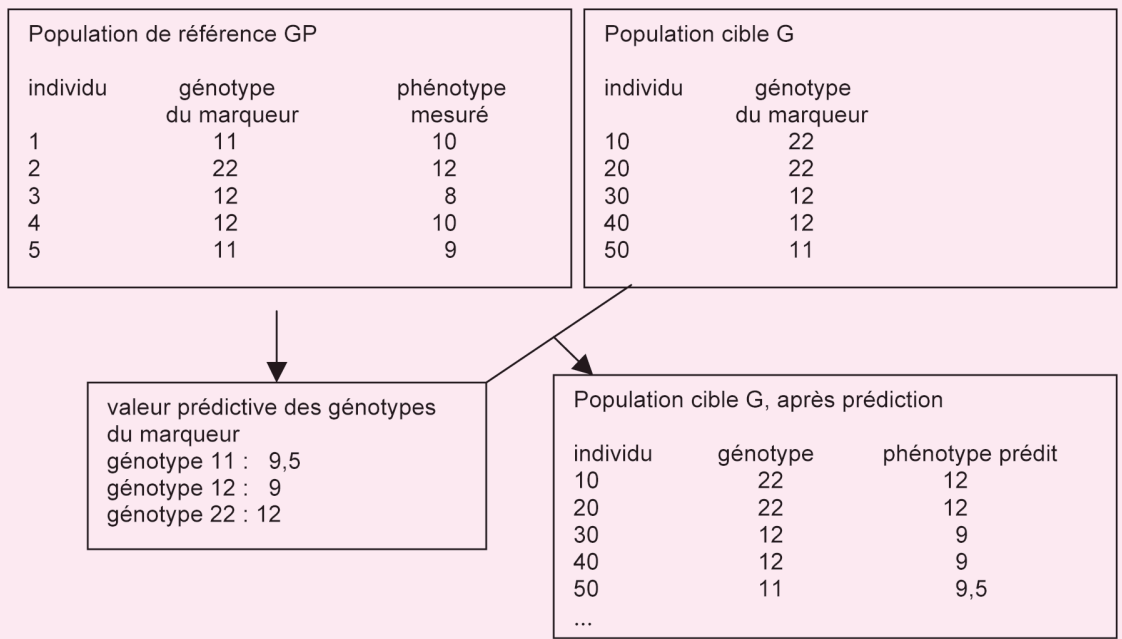

Dans la population GP il est possible d'attribuer des valeurs prédictives à chaque marqueur (1 seul marqueur ici). Dans ce cas d'école, la méthode est la moyenne arithmétique : la valeur pour le génotype 11 est 9,5 soit $(10+9) / 2$. Ensuite, ces valeurs appliquées aux génotypes dans la population $\mathrm{G}$ donnent la prédiction pour les individus de la population cible $\mathrm{G}$ : la prédiction de l'individu 50 est 9,5 car son génotype au marqueur est 11.

Dans les schémas de races traites, plusieurs configurations référence-cible peuvent être envisagées. Trois exemples:

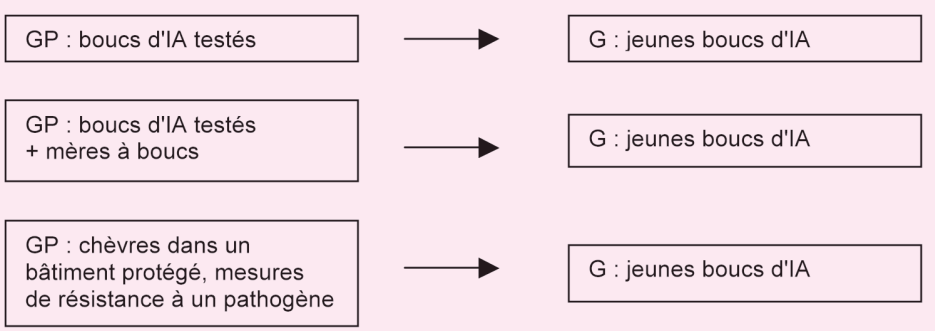

Attention, les index génomiques sont plus fiables quand les animaux des populations $\mathrm{G}$ et GP sont apparentés (Legarra et al 2008).

en ferme. Contrairement aux deux cas précédents, où la population GP était constituée des parents et ancêtres proches de la population $\mathrm{G}$, le cas 3 est sujet à des risques d'incohérence entre les effets des marqueurs estimés dans la population GP et les effets réels des marqueurs dans la population cible $\mathrm{G}$, si les deux populations sont éloignées génétiquement.

A l'heure actuelle, la SAM et la SG sont utilisées dans des schémas de sélection de bovins laitiers, avec l'utilisation de puces à $\mathrm{ADN}$ denses, de 60 à 900000 SNP selon les populations, comme outil moléculaire.

\section{2 / Les programmes d'amélio- ration génétique chez les caprins}

A notre connaissance, la revue faisant l'état des lieux des programmes génétiques en races traites caprines date de la dernière Conférence Mondiale de Génétique Appliquée aux Animaux d'Elevage "WCGALP» à Montpellier en 2002 (Montaldo et
Manfredi 2002), qui elle-même faisait suite à un Symposium Satellite dédié aux programmes de sélection de la Conférence Mondiale Caprine (Tours 2000). Les grandes tendances de ces revues sont toujours d'actualité : la chèvre, en particulier certaines races comme la Saanen, l'Alpine et la Nubienne, connaît une diffusion croissante sur les 5 continents mais les programmes de sélection sont encore rares.

Dans les différents pays ou régions, ces «programmes» ont une complexité croissante, allant de l'organisation pour la vente des reproducteurs jusqu'aux schémas de création de progrès génétique :

- La situation de base est l'existence des associations d'éleveurs pour fixer les objectifs et promouvoir une race locale ou les reproducteurs locaux d'une race internationale. Ces associations sont fréquentes et organisent souvent des registres généalogiques issus des déclarations des saillies et des mise bas. 
- En revanche, le contrôle de performances est beaucoup moins fréquent. Le cas de la France, avec plus de 350000 chèvres contrôlées régulièrement (40\% du cheptel national), est exceptionnel. En effet, parmi la cinquantaine de pays représentés dans l'ICAR $^{8}$ moins de la moitié mentionnent des effectifs en contrôle laitier caprin et ils sont audessous de 30000 chèvres contrôlées annuellement : 28890 chèvres en contrôle en Norvège en 2010 (soit 90\% des élevages), environ 20000 en Espagne, et moins de 5000 dans les autres pays (Montaldo et Manfredi 2002).

- La collecte des performances et des généalogies permet le calcul des index génétiques. Dans les pays qui ont une collecte organisée des données, la calcul régulier des index génétiques caprins sert à comparer les reproducteurs dans la population, surtout quand il y a un minimum de connexions génétiques entre élevages (Fouilloux et al 2008). En France, les index officiels sont calculés pour les caractères laitiers et la morphologie mammaire (encadré 1).

- Les schémas de sélection. A quelques exceptions près comme la France et la Norvège, la situation générale est l'absence de schéma de sélection pour l'ensemble de la population. Aux USA, par exemple, il existe une association de sélectionneurs, l'IA est répandue, un contrôle de performances est mis en œuvre et l'indexation génétique est assurée par l'USDA', mais l'importation et la procréation des reproducteurs sont gérées au niveau du sélectionneur individuel. Cette stratégie en ruminants laitiers, ne permet que des marges réduites de progrès génétique, car les intensités de sélection atteignables sont faibles ${ }^{10}$. L'alternative est la coopération entre sélectionneurs pour partager les reproducteurs et des outils de reproduction. En Norvège (Ådnøy et al 2000), le schéma coopératif est basé sur la monte naturelle alors qu'en France c'est l'IA qui a été privilégiée, comme en bovins et en ovins laitiers. L'encadré 2 illustre le schéma coopératif de sélection des mâles en races Alpine et Saanen. Dans la pratique actuelle, les futurs mâles sont sélectionnés d'abord sur ascendance, via les accouplements programmés entre les meilleurs boucs et les meilleures chèvres de la population, puis sur descendance, par un test basé sur plus de trente filles nées dans plusieurs élevages. L'utilisation des méthodes pour optimiser l'apparentement et la fréquence d'utilisation des reproducteurs permet d'accélérer le progrès génétique tout en limitant à moins de $2 \%$ la consanguinité des jeunes animaux retenus pour renouveler les femelles, les mâles de monte naturelle et les mâles d'IA (Colleau et al 2011).

Le schéma de sélection peut être basé aussi sur la monte naturelle par l'utilisation partagée des boucs entre les éleveurs d'une région. Les "cercles de boucs» mis en place en Norvège dès les années 1970 (Ådnøy et al 2000) permettaient d'organiser le testage des boucs avec un nombre suffisant de filles. Dernièrement, ce schéma a été limité pour des raisons sanitaires, le plan d'éradication du virus CAEV ayant beaucoup limité la circulation des boucs (34 cercles de boucs ont fonctionné jusqu'en 2004 mais seulement 11 cercles subsistent en 2010). En même temps, l'utilisation de l'IA a augmenté grâce à des protocoles simplifiés (Nordstoga et al 2010) qui peuvent être mis en œuvre sans utilisation d'hormones de synchronisation d'œstrus.

Les résultats de ces programmes sont reconnus. En France, ils ont contribué à l'amélioration régulière des performances laitières, d'environ $10 \%$ pour les quantités de matière grasse et protéique durant la dernière décennie, avec une évolution favorable de la richesse des laits (Résultats du contrôle laitier, Institut de l'élevage 2008). Pour ces caractères laitiers, environ la moitié du progrès phénotypique observé peut être attribué à la génétique (Clément et al 2002).

Ces bons résultats, observés sur les $40 \%$ du cheptel français qui sont contrôlés, n'atteignent pas les gains maximum théoriques d'un schéma polygénique appliqué aux ruminants laitiers, soit environ $2 \%$ d'évolution phénotypique par an pour les caractères laitiers. Mais rappelons que la diffusion de l'IA caprine en France est limitée, avec environ 80000 chèvres inséminées/an sur 360000 en contrôle (Leboeuf et al 1998, Leboeuf et al 2008). Côté sélection, l'optimisation d'un schéma combinant l'IA et la monte naturelle permet d'accélérer le progrès (Colleau et al 2011), mais l'accroissement du nombre de candidats à la sélection et la diminution des intervalles entre générations sont également nécessaires pour intégrer efficacement de nouveaux critères de sélection, comme la résistance aux mammites et d'autres caractères à venir, ainsi que pour maîtriser encore mieux la consanguinité.

Avec le schéma actuel, sélection sur ascendance et sur descendance, il faudrait augmenter la taille des séries de testage car un bouc de race laitière n'est candidat qu'après avoir été testé sur descendance. Mais la diffusion actuelle de l'IA ne génère pas les fonds nécessaires pour autofinancer ces tests additionnels sur descendance. On voit bien l'urgence d'avancer vers la sélection génomique dans cette espèce, non pas à cause de la valeur marchande des reproducteurs, mais pour éliminer un coût de testage qui limite l'espace de sélection et qui, en plus, entraîne des intervalles entre générations supérieurs à 5 ans. Rappelons aussi que la production de semence d'un bouc au long de sa carrière est limitée (moins de 10000 doses, en moyenne) et peu rentable par rapport au coût de sa double sélection actuelle, sur ancêtres et sur descendance.

\section{3 / Utilisation des données molé- culaires en sélection caprine : passé et perspectives}

\section{a) Sélection assistée par gènes}

La première SAG dans le schéma caprin était orientée vers l'amélioration de la richesse des laits en utilisant le polymorphisme CSN1S1. Vers 1996, les effets de ce polymorphisme étaient bien connus. A l'époque, l'index génétique était $\mathrm{ICC}=\mathrm{MP}+0,4 \mathrm{TP}$, soit une combinaison linéaire des index «quantité» (MP) et «taux» (TP) des protéines. Les index MP et TP étaient plus élevés pour les boucs porteurs des allèles A (forts) que pour les boucs porteurs des allèles faibles $\mathrm{F}$ ou nuls $\mathrm{O}$. A tel point qu'en race Alpine, la moitié de boucs AA était retenue après testage alors que presque la totalité des boucs homozygotes FF était éliminée au vu de leurs mauvais résultats de testage (Manfredi et al 1995). D'où deux voies d'utilisation potentielles du polymorphisme pour éviter ou limiter la mise en testage de boucs de génotype défavorable (encadré 2) : d'une part, des accouplements complémentaires (parent AA x parent AF, au lieu de AF X AF qui risque de produire des fils FF) et d'autre part, la sélection intra-fratrie des meilleurs génotypes à la naissance des boucs. En pratique, le programme a été limité à la sélection intra-fratrie sans utilisation

\footnotetext{
8 Comité International pour le Contrôle de Performances des animaux en Elevage.

9 Département d'Agriculture des Etats-Unis.

${ }^{10}$ A noter, en revanche, l'existence de firmes de sélection avec des schémas performants en génétique avicole et cunicole, espèces de moindre taille à prolificité élevée et intervalle entre générations faible.
} 
du polymorphisme dans les accouplements programmés (Piacère et al 1997, Manfredi 2003). L'utilisation de ce polymorphisme a été conseillée également en Espagne (Sanchez et al 2005) et a été pratiquée en Norvège pour éliminer les allèles défavorables du gène CSN1S1.

Cette première SAG visait l'amélioration de la valeur génétique moyenne des séries de testage et la diminution de la prise de risque par les éleveurs participant au testage. Comme le polymorphisme était un prédicteur précoce mais partiel (un seul gène) du résultat du test sur descendance, il a été intégré à la sélection sans modification du schéma traditionnel, avec le maintien du testage.

Comme indiqué dans la partie 1 , il y a des allèles de résistance à la tremblante chez les caprins. L'utilisation du polymorphisme du gène $\operatorname{PrP}$ pourrait être envisagée, à l'instar des actions de sélections menées en ovins (Leymarie et al 2009). Mais là encore, il faudrait prévoir des espaces de sélection pour ce nouveau critère de sorte que le progrès génétique sur les autres caractères soit peu dégradé.

\section{b) Possibilités de la Sélection} Génomique

La puce de plus de 50000 SNP (projet CapriSNP) (Palhière et al 2009) couvre l'ensemble du génome, soit toutes les régions inconnues influençant tous les caractères. L'exhaustivité de la SG autoriserait la suppression du test sur descendance et la reformulation du schéma de sélection, comme une première utilisation de la sélection génomique (encadré 2). Par simulation des situations en bovins laitiers, pour un caractère d'héritabilité de 0,2 et des marqueurs expliquant $50 \%$ de la variance génétique totale, Colleau et al (2009) prédisent des progrès génétiques annuels de $0,5 \sigma \mathrm{g}$ en SG contre $0,25 \sigma \mathrm{g}$ en schéma polygénique traditionnel, avec des taux de consanguinité comparables. En pratique, cela se traduirait par l'utilisation optimisée d'un plus grand nombre de reproducteurs, mais avec des coûts réduits par reproducteur. En caprins, le premier écueil est la disponibilité d'une puce à un coût abordable. D'où l'émergence du projet CapriSNP et des collaborations internationales en cours.

L'évolution du taux de diffusion de l'IA détermine la quantité de mâles d'IA et la quantité de leurs filles contrôlées dans la population de référence. Et ces quantités influencent la fiabilité des index génomiques. Le taux a évolué positivement depuis une dizai- ne d'années pour atteindre respectivement 25 et $10 \%$ des chèvres dans la population contrôlée et totale. Le taux d'IA et l'efficacité de la SG sont liés : d'une part, si le taux augmente la SG sera plus efficace, d'autre part, l'implémentation de la SG peut rendre plus attractifs les reproducteurs d'IA (de nouveaux caractères, des précisions d'index améliorées...) et donne des conditions propices pour augmenter la diffusion de l'IA. D'où l'importance d'étudier au moins deux scénarios: taux actuel et taux amélioré de diffusion de l'IA. De même, il conviendra d'étudier des schémas de transition préalables à l'éventuelle suppression du testage. Comme en modèle polygénique, la gestion de la variabilité et l'optimisation des accouplements doivent être considérées dans les scénarios à étudier.

La SG illustrée dans l'encadré 2 est très orientée vers la suppression de test sur descendance. Une réflexion de plus long terme devra aborder d'autres SG possibles : l'évaluation génomique des femelles, la disponibilité d'unités ou des réseaux de phénotypage comme populations de référence (pour les caractères non pertinents dans les routines de contrôle laitier mais qui peuvent être utiles en sélection), la considération des interactions génotypemilieu, ou encore les interactions entre $\mathrm{SG}$ et les techniques de la reproduction.

Les organismes de sélection sont dans une situation privilégiée pour être moteur de tels programmes avec les outils génomiques désormais disponibles. Des collaborations internationales seraient souhaitables pour conforter les populations raciales de référence. Le coût d'un génotypage, inférieur à 200 euros (avec une perspective à la baisse) est bien inférieur au coût de testage actuel. L'hébergement d'un plus grand nombre de reproducteurs peut être raisonné de façon centralisée ou par un réseau d'éleveurs en profitant des structures de coopération déjà existantes.

\section{Conclusion}

Les évolutions de l'amélioration génétique caprine doivent être conçues sur les plans international et multisectoriel, tant pour les objectifs que pour les moyens. En ce qui concerne les objectifs, la demande est de concilier les besoins de la production, du consommateur et de l'impact de la production caprine sur l'environnement. En ce qui concerne les moyens, des collaborations sont en cours à l'échelle inter- nationale pour que cette espèce bénéficie de l'évolution rapide des outils génomiques.

En France, le travail pionnier de l'INRA dans la construction des cartes génétiques caprines (Vaiman et al 1996, Schibler et al 2009) a été relancé pour produire une puce d'environ 50000 SNP dans le projet CapriSNP (Palhière et al 2009). Cet effort est renforcé par des collaborations internationales et la constitution de l'"International Goat Genome Consortium» (http://snp.toulouse.inra.fr/ $\sim$ sigenae $/ 50 \mathrm{~K}$ goat snp c hip/index.html). A moyen terme, une nouvelle génération de séquenceurs, qui devrait rendre les séquences génomiques encore plus accessibles, est attendue.

Pour l'amélioration des productions, les recherches en cours sur la génétique de la résistance aux mammites et l'aptitude à la traite déboucheront sur l'inclusion de ces caractères parmi les critères de sélection. Ce travail sur des caractères d'adaptation des animaux aux contraintes, sera complété par des études déjà envisagées dans le projet européen 3SR sur les caractères de reproduction et sur la détection de polymorphismes influençant la résistance aux infections mammaires et aux parasitismes gastro-intestinaux. Ces caractères contribuent à améliorer le rapport produit/taille du cheptel, comme préconisé par la FAO, pour limiter l'impact des élevages sur l'environnement. Une autre piste à explorer à moyen terme serait de revoir le déterminisme génétique de l'efficacité alimentaire avec une vision génomique de l'animal et de la flore digestive, en interaction avec les régimes alimentaires. Côté consommateur, la diversification des produits caprins reste un objectif, comme c'est le cas au niveau international. Elle est appuyée par des recherches sur la composition en acides gras qui ont commencé dans le projet Phénofinlait (Brochard et al 2009) et par la reprise des études sur la génétique de la qualité diététique des laits caprins. Ces études bénéficient des nouvelles méthodes de phénotypage, comme les nouvelles approches de spectroscopie utilisées pour l'analyse fine des composants du lait (Soyeurt et al 2010, Dagnachew et Ådnøy 2011, Coppa et al 2010).

L'évolution des outils génomiques est également attendue pour améliorer dans le court terme l'efficacité des schémas de sélection. La sélection génomique des caprins laitiers est urgente pour augmenter le rapport bénéfice/coût de la sélection, dans un premier scénario de suppression de la sélection sur descendance. 


\section{Références}

Ådnøy T., Nævdal I., Svendsen M., 2000. Buck circles for dairy goat breeding in Norway. In: World Goat Conf. Satellite Symp. Gruner L., Chabert Y. (Eds). Institut de l'Elevage, INRA, Paris, France, 1047.

Ådnøy T., Vegarud G., Devold T.G., Nordbø R., Colbjørnsen I., Brovold M., Markovic B., Roseth A., Lien S., 2003. Effect of the 0- and F-alleles of alpha S1 casein in two farms of northern Norway. Proc. Int. Workshop Major Genes and QTL in Sheep and Goat. Toulouse, France. On CD-ROM comm. n $2-20,1-5$.

Analla M., Jimenez Gamero I., Munoz Serrano A., Serradilla J.M., Falagan A., 1996. Estimation of genetic parameters for milk yield and fat and protein contents of milk from Murciano-Granadina goats. J. Dairy Sci., 79, 1895-1898.

Asdell S.A., 1944. The genetic sex of intersexual goats and a probable linkage with the gene for hornlessness. Science, 99, 124

Barbieri M.E., Manfredi E., Elsen J.M., Ricordeau G., Bouillon J., Grosclaude F., Mahé M.F., Bibe B., 1995. Effects of the Alpha(s1) casein locus on dairy performances and genetic parameters of Alpine goats. Genet. Sel. Evol., 27, 437-450.

Barillet F., Mariat D., Amigues Y., Faugeras R., Caillat H., Moazami-Goudarzi K., Rupp R., Babilliot J.M., Lacroux C., Lugan S., Schelcher F., Chartier C., Corbière F., Andréoletti O., Perrin-Chauvineau C., 2009. Identification of seven haplotypes of the caprine $\operatorname{PrP}$ gene at codons 127, 142, 154, 211, 222 and 240 in French Alpine and Saanen breeds and their association with classical scrapie. J. Gen. Virol., 90, 769-776.

Bélichon S., Manfredi E., Piacère A., 1999. Genetic parameters of dairy traits in the Alpine and Saanen goat breeds. Genet. Sel. Evol., 31, 529-534.

Bevilacqua C., Martin P., Candalh C., Fauquant J., Piot M., Roucayrol A.M., Pilla F., Heyman M., 2001. Goats' milk of defective \& alpha; s1-casein genotype decreases intestinal and systemic sensitization to beta-lactoglobulin in guinea pigs. J. Dairy Res., 68, 217-227.

Boichard D., Bouloc N., Ricordeau G., Piacère A., Barillet F., 1989. Genetic parameters for first lactation dairy traits in the Alpine and Saanen goat breeds. Genet. Sel. Evol., 21, 205-215.

Brochard M., Faucon F., Barillet F., Bolard M., Brunschwig P., Duhem K., Eggen A., Esvan S., Ferrand M., Fritz S., Gastinel P.L., Guerin J.L., Journaux L., Krychowski T., Lagriffoul G., Larroque H., Lecomte C., Leray O., Leroux C., Leverrier C., Martin P., Mattalia S., Miranda G., Palhière I., Peyraud J.L., Boichard D., 2009. PhenoFinLait: a French national project to detect QTL or major genes affecting the fine composition of dairy ruminants' milk. Renc. Rech. Rum., 16, 423.

Bruckmaier R.M., Ritter C., Schams D., Blum J.W., 1994. Machine milking of dairy goats during lactation - udder anatomy, milking characteristics, and blood-concentrations of oxytocin and prolactin. J. Dairy Res., 61, 457466.

Chanat E., Martin P., Ollivier-Bousquet M., 1999. Alpha-s1-Casein is required for the efficient transport of beta- and kappa-casein from the endoplasmic reticulum to the Golgi appara- tus of mammary epithelial cells. J. Cell Sci., 112, 3399-3412.

Chilliard Y., Rouel J., Leroux C., 2006 Goat's alpha-s1 casein genotype influences its milk fatty acid composition and delta-9 desaturation ratios. Anim. Feed Sci. Technol., 131, 474-487.

Clément V., Boichard D., Piacère A., Barbat A., Manfredi E., 2002. Genetic evaluation of French goats for dairy and type traits. $7^{\text {th }}$ World Conf. Genet. Applied Livest. Prod., Montpellier, France, 1-4.

Clément V., Martin P., Barillet F., 2006 Elaboration d'un index synthétique caprin combinant les caractères laitiers et des caractères de morphologie mammaire. Renc. Rech. Rum., 13, 209-212.

Clément $\mathrm{V}$., Caillat $\mathrm{H}$, Piacère $\mathrm{A}$, Manfredi E., Robert-Granié C., Bouvier F., Rupp R. 2008. Vers la mise en place d'une sélection pour la résistance aux mammites chez les caprins laitiers. Renc. Rech. Rum., 15, 405 408

Colleau J.J., Fritz S., Guillaume F., Baur A., Dupassieux D., Boscher M.Y., Journaux L., Eggen A., Boichard D., 2009. Simulation des potentialités de la sélection génomique chez les bovins laitiers. Renc. Rech. Rum., 16, 419.

Colleau J.J., Clément V., Martin P., Palhière I., 2011. Optimized diffusion of buck semen for saving genetic variability in selected dairy goat populations. BMC Genet., 12.

Coppa M., Ferlay A., Leroux C., Jestin M., Chilliard Y., Martin B., Andueza D., 2010 . Prediction of milk fatty acid composition by near infrared reflectance spectroscopy. Int Dairy J., 20, 182-189.

Cosenza G., Pauciullo A., Colimoro L., Mancusi A., Rando A., Di Berardino D. Ramunno L., 2007. An SNP in the goat CSN2 promoter region is associated with the absence of beta-casein in milk. Anim. Genet., 38, 655658.

Dagnachew B., Ådnøy T., 2011. Genetic variability of goat milk FTIR spectra Husdyrforsøksmøtet, http://www.umb.no/statisk/husdyrforsoksmoter/2011/99.pdf

Fernando R.L., Grossman M., 1989. Marker assisted selection using best linear unbiased prediction. Genet. Sel. Evol., 21, 467-477.

Fevrier C., Jaguelin Y., Lebreton Y., Colleaux Y., Prigent J.P., 2000. Nutritive value for piglets and ileal amino acid digestibility of goat's milk differing in alpha s1 casein. In: World Goat Satellite Symp. Gruner L., Chabert Y. (Eds). Institut de l'Elevage, Paris, France, 876-879.

Folch J.M., Coll A., Sanchez A., 1994. Complete sequence of the caprine BetaLactoglobuli gene. J. Dairy Sci., 77, 34933497.

Fouilloux M.N., Clément V., Laloë D., 2008. Measuring connectedness among herds in mixed linear models: From theory to practice in large-sized genetic evaluations. Genet. Sel. Evol., 40, 145-159.

Furstoss V., David I., Leboeuf B., Guillouet P., Boué P., Bodin L., 2009. Genetic and nongenetic parameters of several characteristics of production and semen quality in young bucks. Anim. Reprod. Sci., 110, 25-36.
Grosclaude F., Martin P., 1997. Casein polymorphisms in the goat. In: Milk protein polymorphism. Proc. IDF Semin., Palmerston North, New Zealand, 241-253.

Grosclaude F., Mahé M.F., Ribadeau-Dumas B., 1973. Structure primaire de la caseine $\alpha$ s1et de la caseine $\beta$-bovine. Eur. J. Biochem. , 40 323-324

Grosclaude F., Mahé M.F., Brignon G., Distasio L., Jeunet R., 1987. A mendelian polymorphism underlying quantitative variations of goat alpha-s1-casein. Genet. Sel. Evol., 19, 399-411.

Hayes B., Hagesæther N., Ådnøy T., Pellerud G., Berg P.R., Lien S., 2006. Effects on production traits of haplotypes among casein genes in Norwegian goats and evidence for a site of preferential recombination. Genetics, 174, 455-464.

Holme I.J., 2009. Estimated variance components for Norwegian dairy goat. Communication personelle. Internal Report of NSG (Norwegian Association of Sheep and Goat Breeders).

Ibeagha-Awemu E.M., Kgwatalala P., Zhao X., 2008. A critical analysis of productionassociated DNA polymorphisms in the genes of cattle, goat, sheep, and pig. Mamm. Genome, 19, 591-617.

Ilahi H., Chastin P., Bouvier F. Arhainx J., Ricard E., Manfredi E., 1999. Milking characteristics of dairy goats. Small Rum. Res., 34 97-102.

Ilahi H., Manfredi E., Chastin P., Monod F., Elsen J.M., LeRoy P. , 2000. Genetic variability in milking speed of dairy goats. Genet. Res., $75,315-319$.

Kennedy B.W., Finley C.M., Bradford G.E., 1982. Phenotypic and genetic relationship between reproduction and milk production in dairy goats. J. Dairy Sci., 65, 2373-2383.

Lamberet G., Degas C., Delacroix-Buche A., Vassal L., 1996. Effect of characters linked to $\mathrm{A}$ and $\mathrm{F}$ caprine alpha(s1)-casein alleles on goat flavour: Cheesemaking with protein-fat exchange. Lait, 76, 349-361.

Lande R., Thompson R., 1990. Efficiency of marker-assisted selection in the improvement of quantitative traits. Genetics, 124, 743-756.

Leboeuf B., Manfredi E., Boué P., Piacère A., Brice G., Baril G., Broqua C., Humblot P., Terqui M., 1998. Artificial insemination of dairy goats in France. Livest. Prod. Sci., 55, 193-203.

Leboeuf B., Delgadillo J.A., Manfredi E., Piacère A., Clément V., Martin P., Pellicer-Rubio M.T., Boue P., de Crémoux R., 2008. Controlling reproduction in selection schemes of dairy goats. INRA Prod. Anim., 21, 391-402.

Legarra A., Robert-Granié C., Manfredi E., Elsen J.M., 2008. Performance of genomic selection in mice. Genetics, 180, 611-618.

Leroux C., Martin P., Mahé M.F., Levéziel H., Mercier J.C., 1990. Restriction fragment length polymorphism identification of goat alpha; s1-casein alleles: a potential tool in selection of individuals carrying alleles associated with a high level protein synthesis. Anim. Genet., 21, 341-351.

Leymarie C., Bouffartigue B., Astruc J.M., Balden M., Barillet F., Bibé B., Bonnot A. 
Boscher M.Y., Bouix J., Brochard M., Dion F., François D., Jouhet E., Julien E., Orlianges M., Moreno C., Plahière I., Perret G., Raoul J., Sidani C., Tiphine L., Raynal A., Bouchel D., Catrou O., Chibon J., Tribon P., 2009. Bilan du programme national d'amélioration génétique pour la résistance à la tremblante du cheptel ovin français. Renc. Rech. Rum., 16, 411-414.

Lu C.D., Potchoiba M.J., Loetz E.R., 1991. Influence of vacuum level, pulsation ratio and rate on milking performance and udder health in dairy goats. Small Rum. Res., 5, 1-8.

Luo M.F., Wiggans G.R., Hubbard S.M., 1997. Variance component estimation and multitrait genetic evaluation for type traits of dairy goats. J. Dairy Sci., 80, 594-600.

Maga E.A., Daftari P., Kültz D., Penedo M.C.T., 2009. Prevalence of alpha-s1-casein genotypes in American dairy goats. J. Anim. Sci., 87, 3464-3469.

Mahé M.F., Manfredi E., Ricordeau G., Piacère A., Grosclaude F., 1994. Effects of the alpha-s1-casein polymorphism on goat dairy performance - A within sire analysis of Alpine Bucks. Genet. Sel. Evol., 26, 151-157.

Manfredi E., 2003. The tale of goat alpha-S1 casein. Proc. Int. Workshop on Major Genes and QTL in Sheep and Goat. INRA, Toulouse, France.

Manfredi E., Ricordeau G., Barbieri M.E., Amigues Y., Bibe B., 1995. Genotype at the alpha(s1)-casein locus and selection of bucks on progeny test in the Alpine and Saanen breeds. Genet. Sel. Evol., 27, 451-458.

Manfredi E., Leboeuf B., Bodin L., Boué P., Humblot P., 1998. Sources de variation génétiques et non génétiques des caractéristiques de produccion de semence chez le bouc. Renc. Rech. Rum., 5, 37-39.

Manfredi E., Piacère A., Lahaye P., Ducrocq V., 2001. Genetic parameters of type appraisal in Saanen and Alpine goats. Livest. Prod. Sci., 70, 183-189

Marnet P.G., Billon P., Da Ponte P., Martin J., Manfredi E., 2001. Aptitude à la traite mécanique chez la chèvre : variabilité génétique et bases physiologiques du débit du lait. Renc. Rech. Rum., 8, 321-327.

Martin P., Grosclaude F., 1993. Improvement of milk protein-quality by gene technology. Livest. Prod. Sci., 35, 95-115.

Mercier J.C., Brignon G., Ribadeau-Dumas B., 1971. Structure primaire de la caseine as 1 bovine. Sequence complete. Eur. J. Biochem., 23, 41-51.

Meuwissen T.H.E., Hayes B.J., Goddard M.E., 2001. Prediction of total genetic value using genome-wide dense marker maps. Genetics, 157, 1819-1829.

Montaldo H., Martinez-Lozano F.J., 1993. Phenotypic relationships between udder and milking characteristics, milk production and california mastitis test in goats. Small Rum. Res., 12, 329-337.

Montaldo H.H., Manfredi E., 2002. Organisation of selection programmes for dairy goats. Proc. $7^{\text {th }}$ World Congr. Genet. Appl. to Livest. Prod., Montpellier, France, Session 1. 1-8.

Naderi S., Rezaei H.R., Pompanon F., Blum M.G.B., Negrini R., Naghash H.R., Balkiz O., Mashkour M., Gaggiotti O.E., Ajmone-Marsan P., Kence A., Vigne J.D., Taberlet P., 2008. The goat domestication process inferred from largescale mitochondrial DNA analysis of wild and domestic individuals. Proc. Nat. Acad. Sci. U.S.A., 105, 17659-17664.

Nordstoga A.B., Soderquist L., Ädnøy T., Farstad W., Paulenz H., 2010. Vaginal deposition of frozen-thawed semen in Norwegian Dairy goats: Comparison of single and double insemination with equal total number of spermatozoa. Theriogenology, 74, 895-900.

Ollier S., Chauvet S., Martin P., Chilliard Y., Leroux C., 2008. Goat's \& alpha; S1-casein polymorphism affects gene expression profile of lactating mammary gland. Animal, 2, 566573.

Pailhoux E., Vigier B., Chaffaux S., Servel N., Taourit S. Furet J.P., Fellous M., Grosclaude F., Cribiu E.P., Cotinot C., Vaiman D., 2001. A 11.7-kb deletion triggers intersexuality and polledness in goats. Nat. Genet., $29,453-458$.

Palhière I., Moreno C., Klopp C., Clément V., Lecomte C., Martin P., Rupp R., Mulsant P., 2009. Détection de SNP dans l'espèce caprine, un premier pas vers la génomique. Renc. Rech. Rum., 16, 421.

Pena R.N., Sanchez A., Folch J.M., 2000 Characterization of genetic polymorphism in the goat beta-lactoglobulin gene. J. Dairy Res., 67, 217-224.

Piacère A., Bouloc-Duval N., Sigwald J.P. Larzul C., Manfredi E., 1997. Utilisation de l'index combiné caprin et du polymorphisme de la caséine alpha s1 dans le schéma de sélection caprin. Renc. Rech. Rum., 4, 187-190.

Remeuf F., 1993. Influence of genetic polymorphism of caprine alpha(s1)-casein on physicochemical and technological properties of goat milk. Lait, 73, 549-557.

Ricordeau G., Bouillon J., Le Roy P., Elsen J.M., 1990. Déterminisme génétique du débit de lait au cours de la traite des chèvres. INRA Prod. Anim., 3, 121-126.

Rønningen R.K., 1965. Causes of variation in the flavour intensity of goat milk. Acta Agric. Scand., 15, 301-342.

Rupp R., Clément V., Piacère A., RobertGranié C., Manfredi E., 2011. Genetic parameters for milk somatic cell score and relationship with production and udder type traits in dairy Alpine and Saanen primiparous goats. J. Dairy Sci., 94, 3629-3634.

Sanchez A., Ilahi H., Manfredi E., Serradilla J.M., 2005. Potential benefit from using the alpha(s1)-casein genotype information in a selection scheme for dairy goats. J. Anim. Breed. Genet., 122, 21-29.

Schibler L. Di Meo G.P. Cribiu E.P. Iannuzzi L., 2009. Molecular cytogenetics and comparative mapping in goats (Capra hircus, $2 n=60)$. Cytogenet. Genome Res., 126, 77-85.

Schmidely P., Meschy F., Tessier J., Sauvant D., 2002. Lactation response and nitrogen, calcium, and phosphorus utilization of dairy goats differing by the genotype for alpha(S1)-casein in milk, and fed diets varying in crude protein concentration. J. Dairy Sci., 85, 2299-2307.

Shrestha J.N.B., Fahmy M.H., 2005. Breeding goats for meat production: a review - 1 Genetic resources, management and breed evaluation. Small Rum. Res., 58, 93-106.

Skjevdal T., 1979. Flavor of goat milk Review of studies on the sources of its variation. Livest. Prod. Sci., 6, 397-405.

Soyeurt H., Misztal I., Gengler N., 2010. Genetic variability of milk components based on mid-infrared spectral data. J. Dairy Sci., 93, 1722-1728.

Vaccari G., Panagiotidis C.H., Acin C., Peletto S., Barillet F., Acutis P., Bossers A., Langeveld J., van Keulen L., Sklaviadis T., Badiola J.J., Andreoletti O., Groschup M. H., Agrimi U., Foster J., Goldmann W., 2009. State-of-the-art review of goat TSE in the European Union, with special emphasis on PRNP genetics and epidemiology. Vet. Res., 40, 40-48.

Vaiman D., Koutita O., Oustry A., Elsen J. M., Manfredi E., Fellous M., Cribiu E.P., 1996. Genetic mapping of the autosomal region involved in XX sex-reversal and horn development in goats. Mamm. Genome, 7, 133-137.

Vaiman D., Schibler L., Bourgeois F., Oustry A., Amigues Y., Cribiu E.P., 1996. A genetic linkage map of the male goat genome. Genetics, 144, 279-305.

Vassal L., Delacroix-Buchet A., Bouillon J., 1994. Effect of AA, EE and FF genetic variants of caprine alpha-s1-casein on cheese yielding capacity of milk and sensory properties of traditional cheese. Lait, 74, 89-103.

Yahyaoui M.H., Pella R.N., Sanchez A., Folch J.M., 2000. Polymorphism in the goat beta-lactoglobulin proximal promoter region. J. Anim. Sci., 78, 1100-1101.

\section{Résumé}

Cette revue bibliographique couvre deux aspects de la génétique des caprins laitiers : la variabilité génétique des caractères et l'amélioration génétique. La majorité des connaissances sur le déterminisme génétique des caractères chez les caprins est issue de l'approche «polygénique» car les outils génériques de la génétique moléculaire sont encore limités dans cette espèce. Toutefois, nous discutons ici surtout les résultats sur quelques gènes bien étudiés en caprins, comme ceux des caséines, de la protéine prion et du cornage. Pour l'amélioration génétique, nous rappelons des principes généraux des méthodes de sélection avec ou sans données moléculaires, avant de discuter les programmes de sélection caprine actuels et leurs perspectives. 


\begin{abstract}
Genetics of Dairy Goats

This review covers two parts of dairy goat genetics: genetic variation of traits and genetic improvement. Most knowledge on the genetic determinism of goat traits comes from polygenic analyses since generic molecular tools have been limited in this species. There are, however, some genes well studied at the molecular level such as milk casein and prion protein genes, whose effects on phenotypes are also discussed. In the genetic improvement chapter, we summarize the general principles of animal breeding, with and without the use of molecular data, before a discussion on the present practices and perspectives of dairy goat selection.
\end{abstract}

MANFREDI E., ÅDNØY T., 2012. Génétique des caprins laitiers. In : Elevage caprin. Baumont R., Sauvant D. (Eds). Dossier, INRA Prod. Anim., 25, 233-244. 